\title{
Embodiment in Virtual Reality for the Treatment of Chronic Low Back Pain: A Case Series
}

This article was published in the following Dove Press journal:

Journal of Pain Research

\author{
Michael S Trujillo (D) \\ Anthony F Alvarez $\mathbb{D}^{\prime}$ \\ Lincoln Nguyen' \\ James Petros ${ }^{1,2}$ \\ 'Department of Research, Karuna Labs, \\ Inc, San Francisco, CA, USA; ${ }^{2}$ Allied Pain \\ and Spine, Los Gatos, CA, USA
}

Purpose: We describe two case studies that use embodiment in virtual reality as a treatment for chronic low back pain. The purpose of this case series was to determine the feasibility of a novel virtual reality-based digital therapeutic for the treatment of chronic pain.

Patients and Methods: Two patients with chronic low back pain received seven sessions, two sessions per week, of a novel digital therapeutic that combines virtual embodiment with graded motor imagery to deliver functional rehabilitation exercises using an off-the-shelf virtual reality system. Pain intensity was measured using a visual analog scale before and after each session to get an indication whether individual sessions of virtual embodiment training decrease pain intensity. Pain catastrophizing scale was assessed before the first session and after the seventh session to determine the extent to which virtual embodiment training can improve psychological symptoms of chronic low back pain.

Results: In both patients, pain intensity was improved after individual sessions of virtual embodiment training as measured by a paired $t$-test: (Patient $\mathrm{A}: \mathrm{t}=2.890, \mathrm{P}<0.05$ ) and (Patient $\mathrm{B}: \mathrm{t}=5.346, \mathrm{P}<0.005$ ). This indicates that individual sessions of virtual embodiment training decrease pain intensity. In both patients, improvements were observed in three subscales of the pain catastrophizing scale (rumination, magnification, and helplessness). This indicates that virtual embodiment training may have benefits for chronic pain symptoms such as pain intensity, pain-related mobility impairment, and disability.

Conclusion: This case series provides evidence that embodiment in virtual reality improves symptoms of persistent chronic low back pain. We propose a mechanism by which virtual embodiment may improve chronic pain symptoms by recontextualizing sensory feedback from the body as patients engage in functional rehabilitation exercises while in virtual reality.

Keywords: centralized pain, digital therapeutics, visual analog scale, pain catastrophizing scale, sensorimotor

\section{Introduction}

Chronic pain is a global healthcare burden with as much as of $11 \%$ of the US population suffering from pain lasting six months or longer ${ }^{1,2}$ and upwards of $20 \%$ in Europe. ${ }^{3,4}$ Chronic pain can lead to depression, anxiety, sleep disturbances, and impairment in cognitive tasks. ${ }^{5}$ Persistent pain poses a substantial societal burden due to impairment of personal wellbeing, lost productivity, and high economic encumbrance of prescription opioid use, abuse, and dependence. It has been reported that health care costs of opioid abuse are as high as $\$ 55$ billion annually in the United States and are expected to grow in the coming years. ${ }^{6}$ Opioids are one of the most common treatment strategies for chronic pain, yet have the potential for
Correspondence: Michael S Trujillo Karuna Labs, Inc., 424B Treat Avenue, San Francisco, CA 94II0, USA

Tel + I 6505809184

Email michael.trujillo@karunalabs.com 
abuse, dependence, misuse, and accidental overdose. ${ }^{7}$ In 2017, US Health and Human Services declared the opioid epidemic a public health emergency due to opioid misuse affecting more than 2 million people and causing more than 47,000 deaths annually. ${ }^{8}$ There is an imperative need for alternative, non-invasive, non-addictive methods for treating chronic pain.

Rapid advances in technologies such as virtual reality (VR) have shown promise for treating a variety of diseases and disorders such as chronic pain. Several recent review publications and meta-analyses have demonstrated the utility and benefits of virtual reality therapies in treating both acute and chronic pain. ${ }^{9-11}$ Established research supports VR as a treatment strategy for burn pain, acute pain, and experimentally induced pain. ${ }^{12-17}$ It has been suggested that the mechanism of action of virtual reality is to influence activation in the anterior cingulate cortex (ACC) and periaqueductal gray (PAG). ${ }^{18}$ Although the precise mechanism of chronic pain remains elusive, Zhou ${ }^{19}$ posited that long-term potentiation (LTP) occurs in the ACC in response to an injury. There is evidence to suggest that the ACC responds to pain with specific rate, spatial, and temporal firing specific to the anticipation of pain. ${ }^{20}$ The ACC sends projections PAG, which is the primary control center for descending pain modulation. LTP in the ACC likely shifts tolerable pain thresholds such that people perceive heightened pain severity (hyperalgesia) and increase the anticipation of a painful state. ${ }^{21}$

Two primary approaches afford analgesic benefits of VR: distraction therapy and immersiveness. ${ }^{9,17,22}$ Distraction therapy temporarily takes attention away from pain while patients are engaged in the VR experience. In immersive VR, the user can interact with an artificial environment to treat chronic pain. One form of immersive VR is virtual embodiment. Virtual embodiment refers to the sense of owning a virtual body, from a firstperson perspective, feeling the agent of the sensory feedback related to the actions of the virtual body. ${ }^{23}$ Through activation of somatosensory and premotor circuitry associated with the body parts that are embodied, ${ }^{24}$ the use of virtual embodiment as an immersive VR technique has been shown to influence pain-free range of motion in patients with unilateral chronic shoulder pain. ${ }^{25}$

The following describes a case series to assess the feasibility of embodiment in VR to exert an effect on the intensity and catastrophizing of chronic low back pain. In these case studies, patients completed seven sessions of a novel virtual reality functional rehabilitation program (Virtual
Embodiment Training ${ }^{\mathrm{TM}}$ (KVET ${ }^{\mathrm{TM}}$ ), Karuna Labs, Inc, San Francisco, CA). Patients successfully completed all planned sessions of KVET ${ }^{\mathrm{TM}}$ without adverse events. Both patients showed improvements on self-rated pain intensity from individual sessions of KVET ${ }^{\mathrm{TM}}$ and improvements on pain catastrophizing after seven sessions.

\section{Materials and Methods}

This study was approved by an Institutional Review Board (Advarra) and all study procedures were conducted in accordance with the principles expressed in the Declaration of Helsinki. Two patients were recruited from local Occupational Health and Pain Clinics that were diagnosed with chronic pain disorders of the lower back with a continual duration of at least six months. Patient A was a 37-year-old male who had been diagnosed with patellofemoral pain syndrome and experienced chronic low back pain for more than seven years. While the specific diagnosis of the chronic low back pain was undetermined, patellofemoral pain syndrome has been shown to correlate with low back pain. ${ }^{26,27}$ Patient B was a 64-year-old male who had experienced chronic low back pain for twenty years. The initial cause of back pain was a workplace injury. Since the injury, Patient $B$ received four back surgeries, including a spinal fusion of L5-S1, a pain pump implant, and a spinal cord stimulator implant. The back surgeries were not effective resulting in a diagnosis of failed back surgery syndrome. Despite multiple interventions, the back pain persisted.

Both patients provided informed consent for the case details to be published and then were screened for exclusion criteria. Although VR has a demonstrated history of safety, it carries the potential for adverse effects. One risk is "simulator sickness," which may involve nausea or dizziness similar to seasickness and can occur when an individual is disoriented by immersive VR. Other, less common side effects include disturbance to balance, eyestrain, blurred vision, fatigue, drowsiness, disorientation, and lack of coordination. Simulator Sickness Questionnaire was administered to both patients. The Simulator Sickness Questionnaire helps to identify the risk of nausea and dizziness in VR. ${ }^{28}$ Neither patient was identified as at risk for simulator sickness.

Patients received seven sessions of KVET ${ }^{\mathrm{TM}}$. KVET ${ }^{\mathrm{TM}}$ sessions were administered on an HTC Vive (HTC Corporation, Bellevue, WA) VR Head-Mounted Display $\left(110^{\circ}\right.$ field of view, $1080 \times 1200$ pixels/eye, $90 \mathrm{~Hz}$ refresh) along with the Vive hand controllers and trackers to provide 
an immersive VR experience. The Vive Tracker can be used to track limb and trunk position and motion while in a VR experience. This setup is paired with a gaming computer with the power to run a VR experience at a high frame rate to mitigate the risk of motion sickness. KVET ${ }^{\mathrm{TM}}$ consist of 30-45-minute sessions of exercises in an embodied VR experience. Virtual embodiment is the first-person perception of sensory feedback related to the actions of a virtual entity, or avatar (See Figure 1). Exercises are designed based on the principles of graded motor imagery (GMI). GMI is a biopsychosocial approach to physical therapy that uses imagery, rehearsal, and simulation to re-learn associations to pain and to improve function. GMI was developed as a gradual reintroduction of motor behavior for individuals with chronic pain and has been demonstrated to be an effective approach to treating Complex Regional Pain Syndrome. ${ }^{29}$

$\mathrm{KVET}^{\mathrm{TM}}$ consists of five exercises delivered through the HTC Vive VR system. Exercises are based on principles of GMI and include motor imagery, graded exposure, and corrective exercises. The motor imagery exercise was a virtual sit-to-stand exercise that required coordination between the trunk and lower limbs. Patients first observed a virtual avatar performing the sit to stand task while the patient maintained a stationary position. The patient was instructed to visualize themselves performing the task while observing the virtual avatar. Following the visualization portion, a chair is placed behind the patient and they are prompted to perform the sit-to-stand movement. The graded exposure exercises consisted of a soccer exercise, a target shooting exercise, and a dish stacking exercise. In the soccer exercise, a soccer ball spawned at predictable locations to the left, in front, and to the right of the virtual avatar. Patients used the HTC Vive hand controllers to embody virtual hands as they attempted to catch or deflect the soccer ball as it was kicked towards a soccer goal. The soccer exercise required coordinated lumbar rotation and flexion to successfully complete the task. Points were awarded for catching or deflecting the soccer ball. In the target shooting exercise, patients used the HTC Vive hand controllers to control a virtual turret gun that shot arrows at targets, requiring patients to combine lumbar rotation and lumbar extension to aim the arrow at and shoot targets. Points were awarded for successfully hitting the targets with the arrows. The dish stacking exercise was a simulated activity of daily living and took place in a virtual kitchen. Patients stacked dishes on predetermined places on a virtual counter using the HTC Vive hand controllers to grasp plates and stack them on the counter using lumbar rotation. The corrective exercise was a virtual ball rolling task where patients embodied a virtual floating platform that tilted in response to patients performing lumbopelvic anterior, posterior, and lateral pelvic tilt. An HTC Vive tracker was secured to patient's lower back to track pelvic tilt motion.

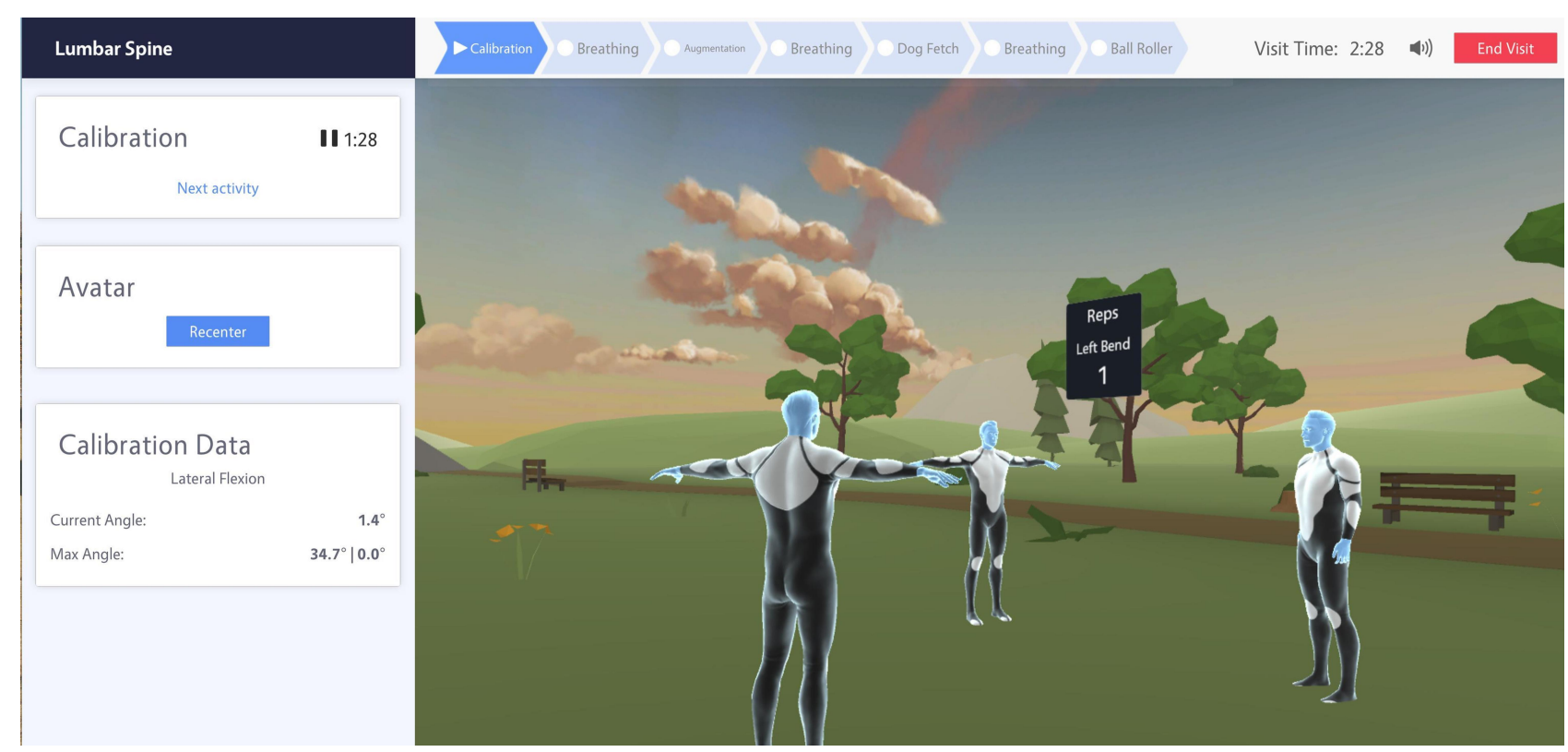

Figure I An example of KVET TM low back exercises. The patient sees the virtual avatar in the first person (front facing forward). A model avatar demonstrates proper lumbar spine exercises which include lumbar flexion, extension, and left and right lateral extension. The avatar to the left produces mirror images of movements performed by the embodied avatar. 
Each session, KVET ${ }^{\mathrm{TM}}$ began with the motor imagery exercise and progressed to the graded exposure exercises followed by the corrective exercise. Each exercise increased in duration as patients became more successful at completing the tasks. Initial sessions were twenty minutes in duration spread out roughly evenly in duration between each exercise. By session seven, total duration was approximately forty-five minutes of $\mathrm{KVET}^{\mathrm{TM}}$ spread evenly between the five exercises.

\section{Results}

Self-perceived pain intensity was evaluated before and after each session of KVETTM using a visual analog scale for pain (VAS). Participants were asked to denote pain using a $10 \mathrm{~cm}$ line with the left end representing no pain and the right indicating the worst possible pain. Participants would then check off the region of the line they believed accurately represented their current pain. The distance between the left most part of the $10 \mathrm{~cm}$ line and the participants check mark was then measured using a standard ruler. This instrument is standard for tracking pain. ${ }^{30}$ A paired $t$-test revealed a significant improvement in pain intensity after single sessions of KVET ${ }^{\mathrm{TM}}$ for Patient A $(\mathrm{t}=2.890, \mathrm{P}<0.05)$ and for Patient $\mathrm{B}(\mathrm{t}=$ $5.346, \mathrm{P}<0.005)$. Pain intensity improved, as measured by a reduction in VAS, in six of seven sessions for Patient A and all seven sessions for Patient B (Figure 2). These results indicate that single sessions of KVET ${ }^{\mathrm{TM}}$ are significant in reducing self-perceived intensity of pain.

To measure catastrophizing, the pain catastrophizing scale (PCS) was administered before the first session of KVET $^{\mathrm{TM}}$ and after the last session. A high level of pain catastrophizing, an excessive negative attending toward pain, has been shown to contribute to the chronicity of low back pain. ${ }^{31}$ The PCS quantifies the negative cognitive and emotional responses to pain. The PCS has three subscales: rumination, magnification, and helplessness. Both Patient A and Patient B improved in all three subscales of PCS (Table 1).

\section{Discussion}

Chronic pain is a life-altering condition resulting in significant reduction in quality of life. ${ }^{32,33}$ Sufferers are often prescribed analgesics, physical therapy, or interventional procedures. These approaches often have incomplete or temporary effects. Opioids are often prescribed but have high potential for abuse, dependence, and overdose, making them a potentially ineffective and dangerous
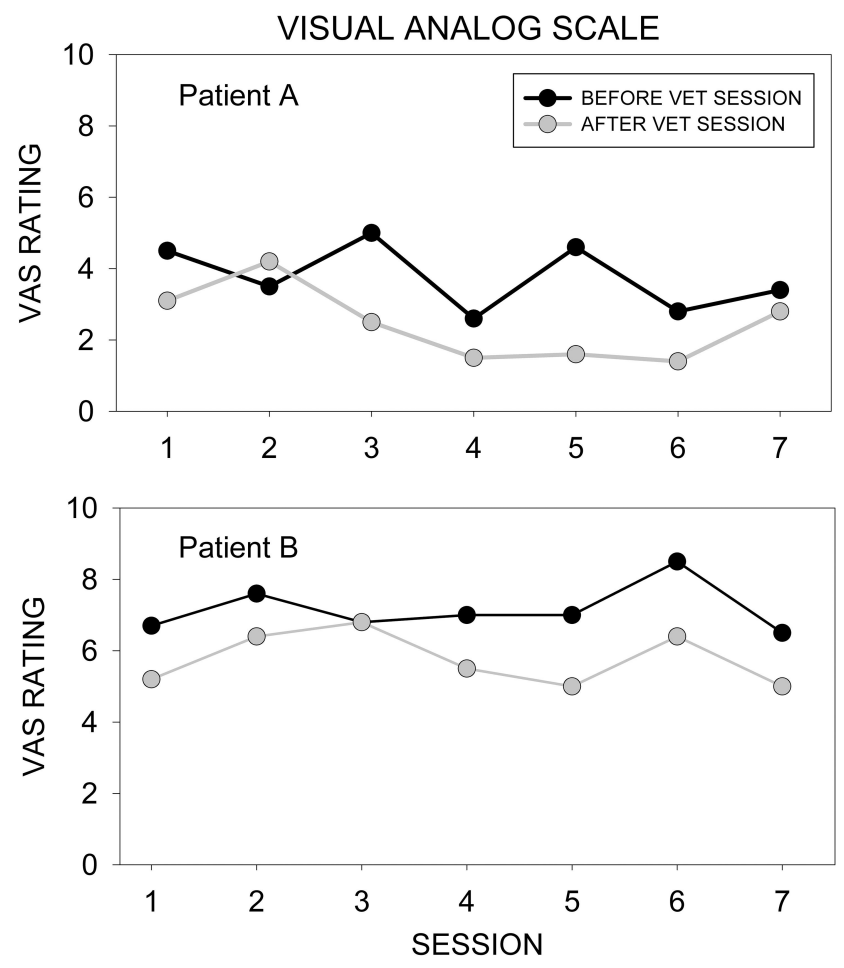

Figure 2 Visual analog scale (VAS) before and after KVET TM for patient A (top) and patient B (bottom).

option. ${ }^{7,34}$ Chronic pain sufferers remain with few efficacious and safe therapeutic options. These case studies provide evidence that virtual embodiment may be a viable treatment strategy.

Chronic pain is often difficult to treat because it is a complex phenomenon that may result from negative neuroplastic mechanisms within the central nervous system that alter the perception of sensory information from the body. ${ }^{19,35-37}$ In patients with a centralized chronic pain mechanism, renormalizing or retraining appropriate sensory processing may improve the symptoms of chronic pain. ${ }^{38}$

Table I Pain Catastrophizing Scale for Subscales Rumination, Magnification, Helplessness, and Total Before the First Session of KVET (Pre) and After the Seventh Session of KVET (Post)

\begin{tabular}{|l|l|l|l|l|}
\hline Subscale & $\begin{array}{l}\text { Pre } \\
\text { (Patient } \\
\text { A) }\end{array}$ & $\begin{array}{l}\text { Post } \\
\text { (Patient } \\
\text { A) }\end{array}$ & $\begin{array}{l}\text { Pre } \\
\text { (Patient } \\
\text { B) }\end{array}$ & $\begin{array}{l}\text { Post } \\
\text { (Patient } \\
\text { B) }\end{array}$ \\
\hline Rumination & 7 & 5 & 11 & 9 \\
Magnification & 3 & 1 & 7 & 3 \\
Helplessness & 9 & 6 & 16 & 10 \\
Total Score & 19 & 12 & 34 & 22 \\
\hline
\end{tabular}

Abbreviations: ACC, anterior cingulate cortex; KVET TM, Karuna Virtual Embodiment Training; PAG, periaqueductal gray; PCS, pain catastrophizing scale; VAS, visual analog scale; VR, virtual reality. 
One of the most effective treatment strategies for chronic pain is psychobehavioral therapy to help patient's cognitive, behavioral, and emotional processes that provoke and reinforce centralized pain symptoms. ${ }^{39-41}$ One of the mechanisms by which VR may be used as a tool to treat chronic pain is the recontextualization of the association between sensorimotor processing of motor behavior. ${ }^{42,43}$ In virtual embodiment, the visual feedback of movement does not come from the patient's own body but from their virtual avatar. Modifications can be made to the movement of the virtual avatar to alter the visual feedback that a patient receives. For example, visual feedback can be augmented, such as overstating or understating the movement of the virtual avatar. If the virtual avatar produces more movement than the patient who is embodied in it, then the patient may perceive that they can move more than they can without inducing a flareup of pain symptoms.

We hypothesize that augmentation of movement in virtual embodiment is the mechanisms that is driving the improvements in self-rated pain intensity and pain catastrophizing. While additional studies would be needed to confirm our hypothesis, previous studies provide evidence that visual feedback augmentation and movement influence pain perception. It has been shown that augmenting visual feedback of a virtual avatar can induce compensation in the movement of an upper extremity limb and induce subconscious motor adaptation. ${ }^{44}$ This suggests that visual feedback of an embodied virtual avatar can influence both physiology and motor behavior even when participants are unaware that movement of the virtual avatar is understated or overstated relative to the participant's own physical movement. Furthermore, greater movement in immersive VR correlates with improved ratings on pain intensity. ${ }^{45}$ Conversely, Gordon et al found that the type of movement of a virtual avatar does not influence pain perception, however, in that study, participants maintained a stationary arm position while observing a virtual arm moving in VR. ${ }^{46}$ It is possible that the lack of physical movement in arm position influenced the sense of immersion or presence in VR. Collectively, these studies suggest that movement combined with immersive VR can influence pain perception. It should be noted that the above studies demonstrated effects on pain perception in a single trial laboratory setting. In the present study, we demonstrate that repeated sessions in immersive VR improve self-rated pain intensity in chronic pain, adding additional evidence that virtual embodiment can be used as a treatment for chronic pain in real-world conditions.
This study demonstrates that immersive VR may have benefits on pain intensity and catastrophizing in chronic low back pain patients, complimenting previous studies that demonstrated benefits of virtual embodiment on chronic neuropathic pain and chronic arm pain, respectively. ${ }^{47,48}$ It has been suggested that improvements in pain catastrophizing has benefits for chronic pain symptoms such as pain intensity, pain-related mobility impairment, and disability. ${ }^{49}$ We hypothesize that the improvements in pain intensity and pain catastrophizing are due to recontextualizing the sensory feedback from the lumbar spine while engaged in functional rehabilitation exercised in virtual embodiment.

Due to the limited number of patients in these case studies, we are not able to draw population-level conclusions of the effect that virtual embodiment may have on chronic pain patients. We confirmed that virtual embodiment is safe, well tolerated, and improves symptoms related to chronic pain on the individual level. Patients also reported that they perceived a benefit from exercises delivered within virtual embodiment. A better powered study in the future is necessary to confirm effects on the population level as well as to determine the durability of virtual embodiment training on symptoms of chronic pain. Additional studies are also needed to determine if different symptoms of chronic pain are influenced by different modalities of GMI and immersive VR. These case studies provide a promising proof-of-concept that virtual embodiment is safe, well tolerated, and improved symptoms in individual chronic low back pain patients.

\section{Conclusion}

The case series described here provide evidence that virtual embodiment can be used to improve pain symptoms in chronic low back pain patients. Individual sessions of virtual embodiment were sufficient in decreasing selfperceived pain intensity and improvements in pain catastrophizing were observed after seven sessions of virtual embodiment training. There are several limitations to this case series. As with all case studies, it is difficult to generalize these results to other chronic pain patients or other chronic pain conditions. However, given the promise of virtual embodiment on improvement on symptoms of chronic pain, additional studies can determine the extent to which immersive VR can alleviate pain and improve function and whether alternate mechanisms beyond visual augmentation can provide similar results or additional benefits. 


\section{Disclosure}

Michael S. Trujillo, Anthony F. Alvarez, and Lincoln Nguyen are employed by Karuna Labs, Inc. James Petros has stock options in Karuna Labs, Inc. The authors report no other conflicts of interest in this work.

\section{References}

1. Johannes CB, Le TK, Zhou X, Johnston JA, Dworkin RH, Solutions RTIH. The prevalence of chronic pain in United States adults: results of an internet-based survey. J Pain. 2010;11 (11):1230-1239. doi:10.1016/j.jpain.2010.07.002

2. Nahin RL. HHS public access. J Pain. 2016;16(8):769-780. doi:10.1016/j.jpain.2015.05.002.Estimates

3. Van Hecke O, Torrance N, Smith BH. Chronic pain epidemiology and its clinical relevance. Br $J$ Anaesth. 2013;111(1):13-18. doi:10.1093/bja/aet123

4. Bouhassira D, Lantéri-minet M, Attal N, et al. Prevalence of chronic pain with neuropathic characteristics in the general population. To cite this version: HAL Id: inserm-00326893 population. Pain. 2008;136(3):380-387. doi:10.1016/j.pain.2007.08.013

5. Apkarian AV, Sosa Y, Krauss BR, et al. Chronic pain patients are impaired on an emotional decision-making task. Pain. 2004;108:129-136. doi:10.1016/j.pain.2003.12.015

6. Leresche L, Turner JA, Saunders K, Shortreed SM, Von Korff M. Psychophysical tests as predictors of back pain chronicity in primary care. J Pain. 2013;14(12):1663-1670. doi:10.1016/j.jpain.2013.08.008

7. Volkow N, McLellan A, Longo DL. Opioid abuse in chronic pain misconceptions and mitigation strategies. $N$ Engl J Med. 2016;374 (13):1253-1263. doi:10.1056/NEJMra1507771

8. Wilson N, Kariisa M, Seth P, Smith H IV, Davis NL. Drug and OpioidInvolved Overdose Deaths — United States, 2017-2018. MMWR Morb Mortal Wkly Rep 2020;69:290-297. doi:10.15585/mmwr.mm6911a4

9. Matamala-gomez M, Donegan T, Bottiroli S, et al. Immersive virtual reality and virtual embodiment for pain relief. Front Hum Neurosci. 2019;13(August):1-12. doi:10.3389/fnhum.2019.00279

10. Malloy KM, Milling LS. The effectiveness of virtual reality distraction for pain reduction: a systematic review. Clin Psychol Rev. 2010;30(8):1011-1018. doi:10.1016/j.cpr.2010.07.001

11. Mallari B, Spaeth EK, Goh H, Boyd BS. Virtual reality as an analgesic for acute and chronic pain in adults: a systematic review and meta- analysis. J Pain Res. 2019;12:2053-2085. doi:10.2147/ JPR.S200498

12. Greenleaf W. How Virtual and Augmented Reality Will Change the Way We Shop. Techno FAQ; 2017.

13. Hoffman HG, Doctor JN, Patterson DR, Carrougher GJ, Furness TA. Virtual reality as an adjunctive pain control during burn wound care in adolescent patients. Pain. 2000;85(1-2):305-309. doi:10.1016/ S0304-3959(99)00275-4

14. Hoffman HG, Seibel EJ, Richards TL, Furness TA, Patterson DR, Sharar SR. Virtual reality helmet display quality influences the magnitude of virtual reality analgesia. J Pain. 2006;7(11):843-850. doi:10.1016/j.jpain.2006.04.006

15. Hoffman HG, Richards TL, Van Oostrom T. The analgesic effects of opioids and immersive virtual reality distraction: evidence from subjective and functional brain imaging assessments. Anesth Analg. 2007;105(6):1176-1783. doi:10.1213/01.ane.0000270205.45146.db

16. Carrougher GJ, Hoffman HG, Nakamura D. The effect of virtual reality on pain and range of motion in adults with burn injuries. J Burn Care Res. 2009;30(5):785-791. doi:10.1097/BCR.0b013e3181b485d3

17. Schmitt YS, Hoffman HG, Blough DK. A randomized, controlled trial of immersive virtual reality analgesia, during physical therapy for pediatric burns. Burns. 2011;37(1):61-68. doi:10.1016/j. burns.2010.07.007
18. Gold JI, Ph D, Belmont KA, Thomas DA, Ph D. The neurobiology of virtual reality pain attenuation. Cyberpsychol Behav. 2007;10 (4):536-545. doi:10.1089/cpb.2007.9993

19. Zhuo M. Long-term potentiation in the anterior cingulate cortex and chronic pain. Philos Trans $R$ Soc Lond B Biol Sci. 2014;369 (1633):20130146. doi:10.1098/rstb.2013.0146

20. Urien L, Xiao Z, Dale J, Bauer EP, Chen Z. Rate and temporal coding mechanisms in the anterior cingulate cortex for pain anticipation. Sci Rep. 2018;8(8298):1-15. doi:10.1038/s41598-018-26518-x

21. Sandkühler J, Gruber-Schoffnegger D. Hyperalgesia by synaptic long-term potentiation (LTP): an update. Curr Opin Pharmacol. 2012;12(1):18-27. doi:10.1016/j.coph.2011.10.018

22. Sharar SR, Carrougher GJ, Nakamura D, Hoffman HG, Blough DK, Patterson DR. Factors influencing the efficacy of virtual reality distraction analgesia during postburn physical therapy: preliminary results from 3 ongoing studies. Arch Phys Med Rehabil. 2007;88 (12SUPPL. 2):43-49. doi:10.1016/j.apmr.2007.09.004

23. Kilteni K, Groten R, Slater M. The sense of embodiment in virtual reality. Presence. 2012;21(4):373-387. doi:10.1162/PRES a 00124

24. Ehrsson HH, Holmes NP, Passingham RE. Touching a rubber hand: feeling of body ownership is associated with activity in multisensory brain areas. J Neurosci. 2005;25(45):10564-10573. doi:10.1523/ JNEUROSCI.0800-05.2005

25. Trujillo M, Alvarez A, Crossland D, Petros J, Nguyen L. Virtual embodiment in virtual reality reveals mirror visual feedback influences on pain-free range of motion. Postgrad Med. 2019;31(S1):64-65.

26. Petersen W, Ellermann A, Gösele-Koppenburg A, et al. Patellofemoral pain syndrome. Knee Surg Sports Traumatol Arthrosc. 2014;22(10):2264-2274. doi:10.1007/s00167-013-2759-6

27. Tsuji T, Matsuyama Y, Goto M, et al. Knee - spine syndrome: correlation between sacral inclination and patellofemoral joint pain. J Orthop Sci. 2002;7(5):519-523. doi:10.1007/s007760200092

28. Kennedy RS, Lane NE, Berbaum KS, Lilienthal MG. Simulator sickness questionnaire: an enhanced method for quantifying simulator sickness. Int J Aviat Psychol. 1993;3(3):203-220. doi:10.1207/ s15327108ijap0303_3

29. Moseley GL. Graded motor imagery is effective for long-standing complex regional pain syndrome: a randomised controlled trial. Pain. 2004;108(1-2):192-198. doi:10.1016/j.pain.2004.01.006

30. Price DD, Mcgrath PA, Rafii A, Buckingham B. The validation of visual analogue scales as ratio scale measures for chronic and experimental pain. Pain. 1983;17(1):45-56. doi:10.1016/0304-3959(83) 90126-4

31. Picavet HSJ, Vlaeyen JWS, Schouten JSAG. Pain catastrophizing and kinesiophobia: predictors of chronic low back pain. Am $J$ Epidemiol. 2002;156(11):1028-1034. doi:10.1093/aje/kwf136

32. Hadi MA, McHugh GA, Closs SJ. Impact of chronic pain on patients' quality of life: a Comparative Mixed-Methods Study. J Patient Exp. 2018;6(2):133-141. doi:10.1177/2374373518786013

33. Elliott TE, Renier CM, Palcher JA. Chronic pain, depression, and quality of life: correlations and predictive value of the SF-36. Pain Med. 2003;4(4):331-339. doi:10.1111/j.1526-4637.2003.03 040.x

34. B artoli A, Kiminek C. What Do The CDC Guidelines Mean for Patients on Long-term High-dose Opioids. Practical Pain Management: Opioid Prescribing and Monitoring. 2017. Available from: https:/www.practicalpainmanagement.com/resource-centers/ opioid-monitoring-2nd-ed/what-do-cdc-guidelines-mean-patientslong-term-high-dose. Accessed August 1, 2020.

35. Phillips K, Daniel CJ. Central pain mechanisms in chronic pain states - maybe it is all in their head. Best Pract Res Clin Rheumatol. 2011;25(2):141-154. doi:10.1016/j.berh.2011.02.005. Central

36. Henry DE, Chiodo AE, Yang W. Central nervous system reorganization in a variety of chronic pain states: a review. Clin Rev. 2011;3 (December):1116-1125. doi:10.1016/j.pmrj.2011.05.018 
37. Latremoliere A, Woolf CJ. Central sensitization: a generator of pain hypersensitivity by central neural plasticity. J Pain. 2009;10 (9):895-926. doi:10.1016/j.jpain.2009.06.012

38. Wälti P, Kool J, Luomajoki H. Short-term effect on pain and function of neurophysiological education and sensorimotor retraining compared to usual physiotherapy in patients with chronic or recurrent non-specific low back pain, a pilot randomized controlled trial. BMC Musculoskelet Disord. 2015;16(1):83. doi:10.1186/ s12891-015-0533-2

39. Darnall BD, Mackey SC, Lorig K, et al. Comparative effectiveness of cognitive behavioral therapy for chronic pain and chronic pain self-management within the context of voluntary patient-centered prescription opioid tapering: the EMPOWER Study Protocol. Pain Med. 2020;21(8):1523-1531. doi:10.1093/pm/pnz285

40. Songer D. Psychotherapeutic approaches in the treatment of pain. Psychiatry. 2005;2(5):19-24.

41. Lumley MA, Schubiner H. Psychological therapy for centralized pain: an integrative assessment and treatment model. Psychosom Med. 2019;81(2):114-124. doi:10.1097/PSY.0000000000000654

42. Riva G, Wiederhold BK, Mantovani F. Neuroscience of virtual reality: from virtual exposure to embodied medicine. Cyberpsychol Behav Soc Netw. 2018;22(1):82-96. doi:10.1089/cyber.2017.29099.gri

43. Wright WG. Using virtual reality to augment perception, enhance sensorimotor adaptation, and change our minds. Front Syst Neurosci. 2014;8:56. doi:10.3389/fnsys.2014.00056
44. Bourdin P, Martini M, Sanchez-Vives MV. Altered visual feedback from an embodied avatar unconsciously influences movement amplitude and muscle activity. Sci Rep. 2019;9(1):19747. doi:10.1038/ s41598-019-56034-5

45. Czub M, Piskorz J. Body movement reduces pain intensity in virtual reality - based analgesia body movement reduces pain intensity in virtual reality - based analgesia. Int J Hum Comput. 2018;1-7. doi:10.1080/10447318.2017.1412144

46. Gordon C, Barbullushi A, Tombolini S, et al. Visuo-tactile stimulation, but not type of movement, modulates pain during the vision of a moving virtual limb. Pain Manag. 2019;9(5):449-460. doi:10.2217/ pmt-2019-0019

47. Pozeg P, Palluel E, Ronchi R, et al. Virtual reality improves embodiment and neuropathic pain caused by spinal cord injury. Neurology. 2017;89(18):1894-1903. doi:10.1212/WNL.0000000000004585

48. Matamala-Gomez M, Diaz Gonzalez AM, Slater M, Sanchez-Vives MV. Decreasing pain ratings in chronic arm pain through changing a virtual body: different strategies for different pain types. J Pain. 2019;20(6):685-697. doi:10.1016/j.jpain.2018.12.001

49. Quartana PJ, Campbell CM, Edwards RR. Pain catastrophizing: a critical review. Expert Rev Neurother. 2009;9(5):745-758. doi:10.1586/ern.09.34

\section{Publish your work in this journal}

The Journal of Pain Research is an international, peer reviewed, open access, online journal that welcomes laboratory and clinical findings in the fields of pain research and the prevention and management of pain. Original research, reviews, symposium reports, hypothesis formation and commentaries are all considered for publication. The manuscript management system is completely online and includes a very quick and fair peer-review system, which is all easy to use. Visit http:// www.dovepress.com/testimonials.php to read real quotes from published authors. 